\title{
Black holes in cascading theories: confinement/deconfinement transition and other thermal properties
}

\author{
Manavendra Mahato and Leopoldo A. Pando Zayas \\ Michigan Center for Theoretical Physics, \\ Randall Laboratory of Physics, The University of Michigan, \\ Ann Arbor, MI 48109-1120, U.S.A. \\ E-mail: mmahato@umich.edu, Ipandoz@umich.edu
}

\author{
César A. Terrero-Escalante \\ Departamento de Física Teórica, Instituto de Física, \\ Universidade do Estado do Rio de Janeiro \\ Maracanã, 20559-900 RJ, Brazil \\ E-mail: cterrero@dft.if.uerj.br
}

\begin{abstract}
We present numerical evidence for a transition between the Klebanov-Strassler background and a solution describing a black hole in the class of cascading solutions in the chirally restored phase. We also present a number of properties of this solution, including the running of the coupling constant, the viscosity to entropy ratio and the drag force on a quark moving in this background.
\end{abstract}

KeYwords: AdS-CFT Correspondence, Black Holes in String Theory, Gauge-gravity correspondence. 


\section{Contents}

1. Introduction 1

2. Review of the cascading black hole 2

2.1 The system of equations from reduction to 1 -d

2.2 Universality of regular nonextremal D3 brane horizons

2.3 Numerics 6

2.4 UV asymptotics and completeness of the solution 0

3. The Hawking-Page transition 10

4. Some properties of a confining field theory at finite temperature via gauge/gravity duality 13

4.1 The running of the gauge couplings at finite temperature 13

4.2 The viscosity bound 15

4.3 Drag force 16

5. Discussion and outlook 20

A. The Klebanov-Strassler background 22

A.1 The action for the KS model 23

A.1.1 The KS background has no regular D3 branes at the apex 25

\section{Introduction}

The AdS/CFT correspondence provides an alternative way to study the strongly coupled regime of gauge theories via gravity duals. The original statement of the AdS/CFT correspondence identifies $\mathcal{N}=4$ supersymmetric Yang-Mills with IIB string theory on $A d S_{5} \times S^{5}$ [1, 2]. At finite temperature the gravity side is described by nonextremal D3 branes and the qualitative matching of the properties was one of the key observations in the understanding and eventual formulation of the AdS/CFT correspondence [3]. Other interesting aspects of finite temperature theories, as seen by the AdS/CFT correspondence were discussed by Witten [四. In particular, the Hawking-Page phase transition in the gravity side was related to the confinement/deconfinement transition on the field theory side.

In a remarkable series of papers [5-9] Klebanov and collaborators carried out a program that concluded with a supergravity background that is dual to a confining field theory with chiral symmetry breaking. This background is known as the warped deformed conifold 
or the Klebanov-Strassler (KS) solution. In the context of the gauge/gravity correspondence it is natural to ask about the possible deconfinement transition for the dual field theory at finite temperature. The finite temperature phase of such theory is certainly very interesting and has been tackled in various papers including [11-13]. In particular, reference [13] constructed a perturbative solution whose regime of validity is restricted to high temperature and small value of the $F_{3}$ flux: $\int_{\Sigma_{3}} F_{3}=P$. Further improvements to this solution were presented in [14]. Knowing the solution only asymptotically in the radial coordinate and for a specific regime of parameters prevents us from extracting the full thermodynamics and from being able to understand possible phase transitions. In particular, in order to answer questions that require knowing the solution for all values of the radial coordinate (for instance, those questions involving the action), it is important to have the full solution which was found numerically in [15].

The thermodynamic aspects of strongly coupled field theories have gained a lot of attention recently, largely stimulated by RHIC. Some of the thermodynamic aspects discussed in this paper have been considered in simpler supergravity backgrounds in [19-23]. The literature is by now very extensive, a representative sample of the different questions that are being pursued can be found in the pages of the Perimeter Institute, where part of our results were preliminarily presented 24 .

The paper is organized as follows. We review the construction of the cascading black hole in section 2. In section 3 we present our main result which is the transition between the KS solution and the cascading black hole. Section 4 discusses various properties of the cascading black hole, including the running of the coupling constant at finite temperature, comments on the viscosity bound and the drag force. We conclude in section 5 with a brief discussion of our main results and some open questions.

\section{Review of the cascading black hole}

To understand the Ansatz for the finite temperature solution we start from the conformal case where the supergravity background is $A d S_{5} \times T^{1,1}$ and the field theory was discussed in [5]. The five dimensional manifold denoted by $T^{1,1}$ is parametrized by coordinates $\left(\psi, \theta_{1}, \phi_{1}, \theta_{2}, \phi_{2}\right)$. The construction of the Ansatz follows directly the one presented originally in [12]. For the metric we consider a generalization consistent with the U(1) symmetry generated by $\psi$-rotations. ${ }^{1}$ The Ansatz in question depends on four functions $(x, y, z, w)$ of the radial coordinate denoted by $u$ :

$$
d s^{2}=e^{2 z}\left(-e^{-6 x} d X_{0}^{2}+e^{2 x} d X_{i} d X^{i}\right)+e^{-2 z} d s_{6}^{2},
$$

where

$$
\begin{aligned}
d s_{6}^{2} & =e^{10 y} d u^{2}+e^{2 y}\left(d M_{5}\right)^{2}, \\
\left(d M_{5}\right)^{2} & =e^{-8 w} e_{\psi}^{2}+e^{2 w}\left(e_{\theta_{1}}^{2}+e_{\phi_{1}}^{2}+e_{\theta_{2}}^{2}+e_{\phi_{2}}^{2}\right) \equiv e^{2 w} d s_{5}^{2} .
\end{aligned}
$$

\footnotetext{
${ }^{1}$ In the gauge theory [5 this symmetry is identified with the $\mathrm{U}(1)_{R}$. Restoring this symmetry at high temperature is understood as chiral symmetry restoration [13]. Therefore, the solution we constructed in 15 corresponds to the deconfined phase due to the presence of a horizon but also to the phase with the chiral symmetry restored due to the fact that $\mathrm{U}(1)_{\psi}$ is a symmetry of the background.
} 
The Funfbein is:

$$
e_{\psi}=\frac{1}{3}\left(d \psi+\cos \theta_{1} d \phi_{1}+\cos \theta_{2} d \phi_{2}\right), \quad e_{\theta_{i}}=\frac{1}{\sqrt{6}} d \theta_{i}, \quad e_{\phi_{i}}=\frac{1}{\sqrt{6}} \sin \theta_{i} d \phi_{i} .
$$

The qualitative meaning of the metric functions $x, y, w$ and $z$ was explained in [12, 15].

The Ansatz for the $p$-form fields is as in the original Klebanov-Tseytlin (KT) solution [8]:

$$
\begin{aligned}
& F_{3}=P e_{\psi} \wedge\left(e_{\theta_{1}} \wedge e_{\phi_{1}}-e_{\theta_{2}} \wedge e_{\phi_{2}}\right) \\
& B_{2}=f(u)\left(e_{\theta_{1}} \wedge e_{\phi_{1}}-e_{\theta_{2}} \wedge e_{\phi_{2}}\right) \\
& F_{5}=\mathcal{F}+* \mathcal{F}, \quad \mathcal{F}=K(u) e_{\psi} \wedge e_{\theta_{1}} \wedge e_{\phi_{1}} \wedge e_{\theta_{2}} \wedge e_{\phi_{2}} .
\end{aligned}
$$

Note that the form of $F_{3}$ is such that it describes a constant flux along a 3-cycle, that is, $\int_{\Sigma_{3}} F_{3}=P$. In some other notation this is called the number of fractional D3 branes. The Bianchi identity for the 5-form, $d * F_{5}=d F_{5}=H_{3} \wedge F_{3}$, implies

$$
K(u)=Q+2 P f(u)
$$

where $Q$ is a constant. $P$ and $Q$ are known as $M$ and $N$ in the standard literature (see review [10]).

Thus, in the presence of 3 -form flux $(P \neq 0)$, the flux of $F_{5}$ varies with the radius. The fact that $K(u)$ depends on the coordinate $u$ is very novel and has interesting physical implications. Phenomenologically, a very attractive property of this class of supergravity solutions is that it encodes the logarithmic running of a combination of gauge couplings in field theory. It does so via a varying $B_{2}$ field which is compensated by a constant $F_{3}$ flux through a 3 -cycle. The five-form, which is constant in most solutions, varies according to the Bianchi identity $d F_{5}=H_{3} \wedge F_{3}$ and generates a varying flux as $\int_{\Sigma_{5}} F_{5}$ depends on the radial coordinate. The supergravity solution therefore has varying flux. This varying flux was interpreted in [9] as the dual of a Seiberg duality cascade, coining therefore the term of cascading solutions.

\subsection{The system of equations from reduction to 1 -d}

An efficient way to derive the system of type IIB supergravity equations of motion is to start with the 1-d effective action for the radial evolution which follows from the 10-d action. This approach is convenient because to establish the transition we compare the actions. So, a simple action is an advantage.

The type IIB supergravity equations of motion follow from the action

$$
\begin{aligned}
S_{10}= & -\frac{1}{2 \kappa_{10}^{2}} \int d^{10} x\left(\sqrt { - g _ { 1 0 } } \left[R_{10}-\frac{1}{2}(\partial \Phi)^{2}-\frac{1}{12} e^{-\Phi}\left(\partial B_{2}\right)^{2}\right.\right. \\
& \left.\left.-\frac{1}{2} e^{2 \Phi}(\partial \mathcal{C})^{2}-\frac{1}{12} e^{\Phi}\left(\partial C_{2}-\mathcal{C} \partial B_{2}\right)^{2}-\frac{1}{4 \cdot 5 !} F_{5}^{2}\right] \frac{1}{-2 \cdot 4 ! \cdot(3 !)^{2}} \epsilon_{10} C_{4} \partial C_{2} \partial-B_{2}+\ldots\right), \\
& \left(\partial B_{2}\right)_{\ldots}=3 \partial_{[.} B_{. .]}, \quad\left(\partial C_{4}\right)_{\ldots .} \equiv 5 \partial_{[\cdot .} C_{\ldots .}, \quad F_{5}=\partial C_{4}+5\left(B_{2} \partial C_{2}-C_{2} \partial B_{2}\right),
\end{aligned}
$$


supplemented with the on-shell constraint $F_{5}=* F_{5}$.

For the metric (2.1)

$$
\begin{gathered}
\sqrt{G}=\frac{1}{108} e^{10 y-2 z} \sin \theta_{1} \sin \theta_{2} \\
\int d^{10} x \sqrt{G} R \rightarrow C_{b . h .} \frac{1}{27} \int d u\left[5 y^{\prime 2}-3 x^{\prime 2}-2 z^{\prime 2}-5 w^{\prime 2}+e^{8 y}\left(6 e^{-2 w}-e^{-12 w}\right)\right],
\end{gathered}
$$

where $C_{b . h .}=64 \pi^{3} \beta_{b . h .} V_{3}$. The inverse temperature of the black hole is represented by $\beta_{b . h}$. and $V_{3}$ is the spatial volume in the $x^{1}, x^{2}$ and $x^{3}$ directions.

The matter part is

$$
\begin{aligned}
& \int d^{10} x \sqrt{G}\left[-\frac{1}{2}(\partial \Phi)^{2}+\ldots\right] \rightarrow \\
& -C_{b . h .} \frac{1}{27} \int d u \frac{1}{8}\left[\Phi^{\prime 2}+2 e^{-\Phi+4 z-4 y-4 w} f^{\prime 2}+2 e^{\Phi+4 z+4 y+4 w} P^{2}+e^{8 z}(Q+2 P f)^{2}\right] .
\end{aligned}
$$

The 1-d effective lagrangian is:

$$
\begin{aligned}
L & =T-V, \\
T & =5 y^{\prime 2}-3 x^{\prime 2}-2 z^{\prime 2}-5 w^{\prime 2}-\frac{1}{8} \Phi^{\prime 2}-\frac{1}{4} e^{-\Phi+4 z-4 y-4 w} f^{\prime 2}, \\
V & =-e^{8 y}\left(6 e^{-2 w}-e^{-12 w}\right)+\frac{1}{4} e^{\Phi+4 z+4 y+4 w} P^{2}+\frac{1}{8} e^{8 z}(Q+2 P f)^{2},
\end{aligned}
$$

supplemented with the "zero-energy" constraint $T+V=0$.

This is effectively a classical mechanical system. The simplest equation is for the nonextremality function $x$ (note that it breaks Loretz invariance in the $\left(X^{0}, X^{1}, X^{2}, X^{3}\right)$ plane):

$$
x^{\prime \prime}=0, \quad x=a u, \quad a=\text { const. }
$$

The reason for such a simple equation is that, as explained in 12], it does not appear in the effective one dimensional Lagrangian, except for its kinetic term.

The other functions $y, w, z, f$ and $\Phi$ should satisfy a coupled system of equations:

$$
\begin{aligned}
10 y^{\prime \prime}-8 e^{8 y}\left(6 e^{-2 w}-e^{-12 w}\right)+\Phi^{\prime \prime} & =0, \\
10 w^{\prime \prime}-12 e^{8 y}\left(e^{-2 w}-e^{-12 w}\right)-\Phi^{\prime \prime} & =0, \\
\Phi^{\prime \prime}+e^{-\Phi+4 z-4 y-4 w}\left(f^{\prime 2}-e^{2 \Phi+8 y+8 w} P^{2}\right) & =0, \\
4 z^{\prime \prime}-(Q+2 P f)^{2} e^{8 z}-e^{-\Phi+4 z-4 y-4 w}\left(f^{\prime 2}+e^{2 \Phi+8 y+8 w} P^{2}\right) & =0, \\
\left(e^{-\Phi+4 z-4 y-4 w} f^{\prime}\right)^{\prime}-P(Q+2 P f) e^{8 z} & =0 .
\end{aligned}
$$

The solutions to this system are subject to the zero-energy constraint $T+V=0$, i.e.

$$
\begin{aligned}
& 5 y^{\prime 2}-2 z^{\prime 2}-5 w^{\prime 2}-\frac{1}{8} \Phi^{\prime 2}-\frac{1}{4} e^{-\Phi+4 z-4 y-4 w} f^{\prime 2}-e^{8 y}\left(6 e^{-2 w}-e^{-12 w}\right) \\
&+\frac{1}{4} e^{\Phi+4 z+4 y+4 w} P^{2}+\frac{1}{8} e^{8 z}(Q+2 P f)^{2}-3 a^{2}=0
\end{aligned}
$$


This constraint was used in [15] as a criterion for quality of the numerical solution and it was established that for various known solutions it was of the order $10^{-16}$ where the reliable accuracy was shown to be $10^{-10}$ (see figure 11 and section 2.3 for a detailed description).

The function $y$ amounts to a choice of the radial coordinate. This is relevant for understanding the dimensions of all quantities. Note that in particular, the system (2.12) and the constraint (2.13) are invariant under $e^{y} \rightarrow L_{0} e^{y}$ and $u \rightarrow L_{0}^{-4} u$ if we assume that $Q \rightarrow L_{0}^{4} Q, P \rightarrow L_{0}^{2} P, a \rightarrow L_{0}^{4} a$ and $f \rightarrow L_{0}^{2} f$. We therefore express all dimensionfull quantities in units where $L_{0}=1$.

Standard regular non-extremal D3-brane solution. To develop our intuition, we present the nonextremal D3 brane of the system (2.12) in the radial $u$-coordinate. It takes the following form:

$$
e^{4 y}=\frac{a}{\sinh 4 a u}, \quad e^{4 z}=\frac{a}{q \sinh 4 a u}, \quad e^{4 x}=e^{4 a u} .
$$

Note that near the horizon $(u \rightarrow \infty)$

$$
\begin{aligned}
y & =y_{*}-a u+\frac{1}{4} e^{-8 a u}+O\left(e^{-16 a u}\right), & z & =z_{*}-a u+\frac{1}{4} e^{-8 a u}+O\left(e^{-16 a u}\right), \\
y_{*} & =\frac{1}{4} \ln 2 a, & z_{*} & =\frac{1}{4} \ln \frac{2 a}{q},
\end{aligned}
$$

A more recognizable form of this solution is given by:

$$
\begin{aligned}
d s^{2} & =h^{-1 / 2}\left(g d X_{0}^{2}+d X_{i} d X_{i}\right)+h^{1 / 2}\left[g^{-1} d \rho^{2}+\rho^{2}\left(d M_{5}\right)^{2}\right], \\
g & =e^{-8 x}=1-\frac{2 a}{\rho^{4}}, \quad \rho^{4}=\frac{2 a}{1-e^{-8 a u}}, \quad h=e^{-4 z-4 x}=\frac{q}{\rho^{4}} .
\end{aligned}
$$

Let us clarify the relationship between the radial coordinate $u$ and the more standard coordinate $\rho$. Using $2 a=\rho_{0}^{4}$, we have

$$
d u=\frac{d \rho}{\rho^{5}}\left(1-\frac{\rho_{0}^{4}}{\rho^{4}}\right)^{-1} .
$$

This can be integrated to

$$
u=-\frac{1}{4 \rho_{0}^{4}} \ln \left(1-\frac{\rho_{0}^{4}}{\rho^{4}}\right) .
$$

Note that in the domain of $\rho_{0} \leq \rho<\infty$ we have that $u$ ranges in $0<u<\infty$. For large values of $\rho$ we have that $u \approx 1 / 4 \rho^{4}$. The position of the horizon which is finite in the $\rho$ coordinate becomes infinite in the $u$ coordinates.

\subsection{Universality of regular nonextremal D3 brane horizons}

For the general solution of the system (2.12), in the $u$-coordinates the area of a surface defined by a horizon at $u=$ constant is

$$
A=V \omega_{5} \exp (-2 z+3 x+5 y) .
$$


Given that the equation of motion for $x$ has the general solution $x=a u$ we are forced into the following situation. If the horizon is at $u \rightarrow \infty$, as is the case for the non-extremal D3 brane solution, then for the area $A$ to be finite we need the following asymptotics for $z$ and $y$ :

$$
z \rightarrow \alpha a u+z_{*}, \quad y \rightarrow \beta a u+y_{*},
$$

with the condition that

$$
3-2 \alpha+5 \beta=0 .
$$

Note that the regular nonextremal D3 brane corresponds to $\alpha=\beta=-1$. The main claim is that: The existence of a regular horizon fixes the asymptotic behavior of the metric coordinates $x, y$ and $z$ near the horizon. eq. (2.23) was used in [15] as a criterion of quality of the numerical solution near the horizon (see table 1 for the precision of this criterion for the solutions we discuss here).

Similarly, one can obtain an expression for the temperature. Namely, the relevant part of the metric is

$$
d s^{2}=e^{2 z-6 x} d \tau^{2}+e^{-2 z+10 y} d u^{2} .
$$

We introduce a new radial coordinate as:

$$
\rho=e^{z-3 x}
$$

We can now rewrite the metric as:

$$
d s^{2}=\frac{e^{-4 z+10 y+6 x}}{\left(z^{\prime}-3 x^{\prime}\right)^{2}}\left[d \rho^{2}+\rho^{2}\left(e^{2 z-5 y-3 x}\left(z^{\prime}-3 a\right) d \tau\right)^{2}\right] .
$$

Note that, again, the requirement of finite temperature fixes the large-u asymptotic of various metric functions to be $2 z-5 y-3 x \rightarrow$ constant. Requiring the absence of conical singularity we find that the temperature defined as the inverse of the period is

$$
T=\frac{|\alpha-3|}{2 \pi} a e^{2 z_{*}-5 y_{*}}
$$

where we used that near the horizon the asymptotic form of $z$ and $y$ is given by (2.22).

\subsection{Numerics}

In ref. [15] we gave a detailed description of the construction of solutions to the system (2.12) which contain regular black holes in the infrared (large values of $u$ ) and a cascading behavior in the ultraviolet (small values of $u$ ). We will summarize here our approach. For more details on the numerical method and the understanding of the numerical output, we refer the interested reader to ref. [15].

To solve the equations for the metric functions we use the seventh-eight order continuous Runge-Kutta method. Thanks to its adaptive scheme, this method provides a great control upon the output accuracy. We tested and fitted the numerical procedure using a number of known analytical solutions, some of them with fixed values of the parameters (for instance, the non-extremal D3 black hole, with $P=0$, and the KT background, with 


\begin{tabular}{|c|c|c|c|c|c|}
\hline$a$ & $P$ & $u_{90 \%}$ & $3-2 \alpha+5 \beta$ & $u_{\text {sing }}$ & $f_{1}+P \mathrm{e}^{\phi_{0}+4 y_{0}+4 w_{0}}$ \\
\hline 1 & 4.26906025 & 2.19361453 & $-9 \times 10^{-15}$ & $3 \times 10^{-10}$ & 0.00001257 \\
\hline 0.5 & 2.87343889 & 4.387229 & $-7 \times 10^{-15}$ & $3 \times 10^{-11}$ & 0.0000065 \\
\hline 0.1 & 1.094389139 & 21.9361453 & $-2 \times 10^{-15}$ & $4 \times 10^{-10}$ & 0.00000002 \\
\hline 0.01 & 0.316430028 & 219.361453 & $10^{-15}$ & $2 \times 10^{-9}$ & 0.0000000002 \\
\hline
\end{tabular}

Table 1: Values of the parameters used for the four cases presented in this manuscript. $Q=L_{p}=1$ was used everywhere.

$a=0$ ). Analyzing the sensitivity of the solutions to the variation of these parameters, we found that for a method tolerance of $10^{-14}$, an error of $10^{-10}$ can be safely regarded to be negligible, what allowed us to set our numerical 'zero' to this last value.

Using the non-extremal D3 solution $(P=0)$, we set the boundary conditions for our ten variables at the value of $\mathrm{u}$ corresponding to the $90 \%$ of the distance to the horizon. A correction is introduced to enforce the solution with $P \neq 0$ to satisfy constraint (2.13) in this boundary.

Next we integrate backward and, for a given value of $a$ and $Q$, we find the value of $P$ such that the space is complete, i.e., $u_{\text {sing }} \approx 0$ (see section 2.4 and table 1). We use

$$
f_{1}+P \mathrm{e}^{\Phi_{0}+4 y_{0}+4 w_{0}}=0
$$

to check that this singularity is of the type given by

$$
z=-\frac{1}{4} \ln \left[4\left(u-u_{\text {sing }}\right)\right]
$$

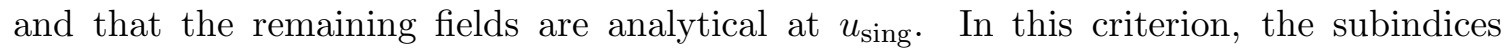
indicates that these are coefficients in Taylor series for the corresponding field at $u_{\text {sing. }}$.

We then integrate the system forward and identify numerically the presence of a horizon. One of the criteria used is that given by eq. (2.23).

In table 11 we present the values of the parameters and the results for the infrared and ultraviolet criteria of quality for four cases that are going to be used as examples throughout this manuscript.

We also recall that system (2.12) was modified in such a way that the solutions must automatically satisfy constraint (2.13). This allows to use the constraint as a dynamical criterion of the quality of the numerical output for any value of the parameters. Indeed, all our solutions are warranted to satisfy constraint (2.13) within the accuracy given by our numerical 'zero'. This can be verified in figure 1. For all the four cases in the table, the $T+V=0$ constraint is satisfied in the whole range of the radial coordinate.

\subsection{UV asymptotics and completeness of the solution}

Let us first discuss the completeness of our solution. The statement that completeness of the space requires $u_{\text {sing }}=0$ was made explicitly in 15] (see section 4.2 there), however, the 


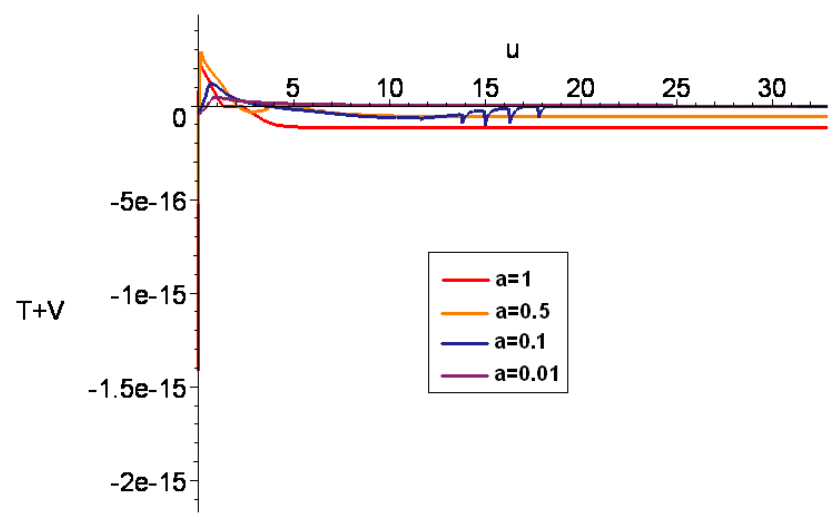

Figure 1: The Hamiltonian constraint for the cases in table 1 .

details were not shown. Here we provide those details since they are crucial in clarifying the number of parameters of the solution. ${ }^{2}$

The asymptotic form of the relevant part of the metric, as obtained in [15], is given for $z(u)$ by eq. (2.29) and for $y(u)$ by $\exp (4 y)=1 / 4 u$. The first expression was widely discussed in [15] and we refer the reader to that paper for details, including the fittings. The expression of $y$ is ubiquitous in the region of small values of $u$. It is valid in the asymptotic region for all the solutions we know: KW [5], nonextremal D3 branes 12, KT [8] and KS [9]. The best fit analysis presented in [15] indicates that this seems to be also the case for our numerical solution.

To discuss completeness we consider an outgoing null geodesic toward the asymptotic regions of small $u$. The corresponding effective Lagrangian is:

$$
\mathcal{L}=-e^{2 z-6 x} \dot{t}^{2}+e^{-2 z+10 y} \dot{u}^{2}
$$

With this information the affine time for a geodesic to reach $u_{\text {sing }}$ satisfies:

$$
d \lambda=\frac{1}{E} u^{-5 / 4} d u
$$

Note that this is independent of the precise form of the $z$ function in the metric. The structure of the function $z$ enters only through the integration limits. Namely, the above equation can be integrated to yield

$$
\lambda=\frac{4}{E}\left(\frac{1}{u_{\text {sing }}}-\frac{1}{u_{\text {in }}}\right) .
$$

Here $u_{\text {in }}$ should be viewed as a cutoff, beyond which we do not know the precise formula for $y$. However, we assume that this geodesic originates somewhere near the black hole

\footnotetext{
${ }^{2}$ This point has been an important source of discussion with A. Buchel and we hope that this section clarifies that $u_{\text {sing }}=0$ fixes one of the three seemingly free parameters $(Q, P, a)$.
} 
horizon. Note that the value $u_{\text {sing }}$ enters just through the integration limits, since the full

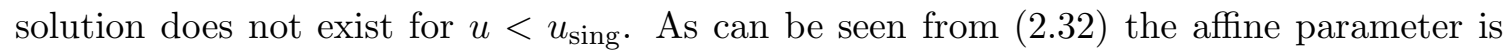
infinite only for $u_{\text {sing }}=0$. We need an infinite affine parameter to guarantee completeness of the solution. Thus, the solution is complete only for $u_{\text {sing }}=0$.

The number of relevant parameters. Thus, superficially, our solutions depend on three parameters, $a, P$ and $Q$. However, the condition $u_{\operatorname{sing}}(\cdots ; a, P, Q)=0$ imposes a new constraint on the solutions that effectively reduces the number of parameters to two.

For instance, fixing $Q=1$, for the values of $a$ given in table $\mathbb{1}$, it is found that the expression,

$$
u_{\text {sing }}=b^{-P^{2}}+b_{0}+b_{1} P+b_{2} P^{2}+\cdots,
$$

fits very well the numerical output. Note that the first term is clearly related to the strong energy scale as indicated by the KT formula, that is, it is related to the radial position where the KT warp factor vanishes; of course, there are many corrections as the KT warp factor is not the full answer. To illustrate the origins and robustness of the expression (2.33), let us first discuss a specific point in the graph 2 to highlight the importance of the first term in the above expansion. Namely, for $a=0.5$, a best fit to the value of $u_{\text {sing }}$ without the exponential term (i.e., setting $\mathrm{b}=0$ ) yields the following result for the coefficients $b_{i}$ :

$$
\begin{array}{r}
\{0.05854539,-0.25108172,0.14454983,-0.03078276, \\
\left.0.00347435,-0.00020362,0.488 \times 10^{-5}\right\}
\end{array}
$$

with a goodness of fit on the order of $10^{-7}$. It is important to stress that for each term we verify that $b_{i} \leq b_{i+1}$, which indicates convergence. Now, introducing the exponential term, the result is $b=5.49169633$,

$$
\begin{array}{r}
\{-0.09506874,-0.09298412,0.07882655,-0.01663906, \\
\left.0.00180981,-0.00010186,0.234 \times 10^{-5}\right\},
\end{array}
$$

with a goodness of fit on the order of $10^{-9}$. The ratio $b_{i} / b_{i+1}$ is also much better behaved. In general the exponential term seems to be crucial for the form of $u_{\text {sing. Thus, for }(a, Q)}$ fixed, by setting $u_{\text {sing }}=0$ in expression (2.33), we are this way selecting a given value of $P$. A similar analysis was carried out for the other values of $a$ in table 1 and another ten cases not included in the table but shown as crosses in figure 2. The curve in this figure represents the pairs $(a, P)$ that guarantee $u_{\text {sing }}$ to vanish for $Q=1$. It was obtained as the best fit solution,

$$
P=4.26846923 a^{0.59296839}+0.02429978
$$

with a goodness of fit of 0.00124 , which is satisfactory if the small amount of data used is taken into account.

Summarizing, for any values of two of the parameters determining our solution, the value of the third one is fixed by imposing completeness of the solution in the asymptotically UV region. 


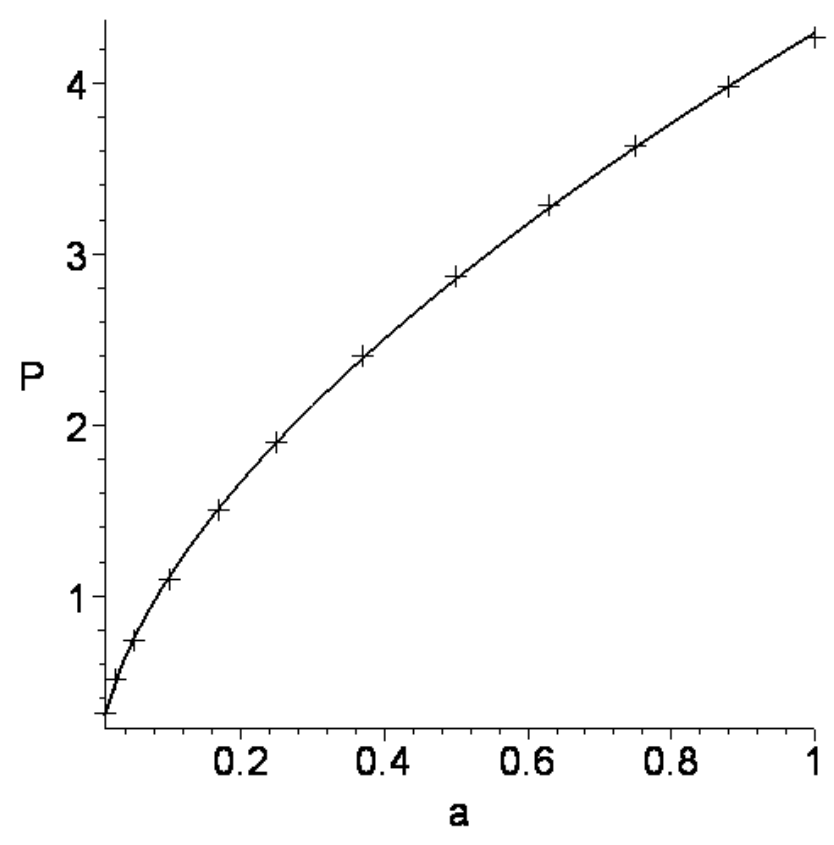

Figure 2: Values of $P$ and $a$ that guarantee $u_{\text {sing }}$ to vanish for $Q=1$.

\section{The Hawking-Page transition}

There is mounting evidence for the transition we are going to describe here. In particular, similar transitions have been established in some simpler holographic models, including [29-32].

In our case, we assume that the deconfined phase is dual to the cascading black hole reviewed in the previous section, while the confined phase is dual to the warped deformed conifold, i.e., the Klebanov-Strassler background [9]. The relevant expressions of this well known solution were compiled in the appendix in terms of the coordinates and notation we use in this paper.

To accurately compute the actions we used an adaptive 3-5 Simpson's quadrature. This implies $2^{n-1} 8^{n}$ evaluations of the Lagrangian, where $n$ is the number of iterations needed to reach the desired precision (set here to $10^{-4}$ ) for the integral. For the cascading black hole the Lagrangian is given in terms of the numerical solutions of system (2.12). We gave priority to the output accuracy over the computational effort. This means being able to obtain the actual numerical solution of (2.12) at every point required by the quadrature, instead of finding it by interpolating between previously calculated solutions at a given set of points. The whole computation of the cascading black hole action usually takes more than 12 hours in an ordinary desktop computer. Compared with that, estimating the action of the KS background is a quick task, since the evaluation of the corresponding Lagrangian only involves the numerical integration of (A.14) which for large $\tau$ can be accurately estimated using expression (A.17). Taking the above into account, we decided to compute the cascading black hole action for the four sets of parameters given in table 1 and compare the results with the output of the KS action as we vary $\epsilon$. 
So, we calculated the cascading black hole action as given by eqs. (2.7) and (2.8), without considering the numerical factors common to both actions. The integration was done between $u_{R}=10^{-8}$ and $u_{90 \%}$. Following the order in table 1, the four results are $\{738451,762361,733711,804269\}$, and their logarithms are shown in figure 4 as horizontal lines. To leading order the asymptotic values of the action are independent of $a$. This can be seen by directly evaluating the pieces of the action as given in (2.7) and (2.8). For example, the leading contribution from (2.7) comes from $z^{\prime 2}$ and after the integration is proportional to $1 / u_{R}$. Similarly, from the matter part (2.8), the leading contribution comes from the last term which is independent of $a$; this term also contributes a term proportional to $1 / u_{R}$. Note that in terms of the standard radial coordinates, this corresponds to $R^{4}$ where $R$ is a UV cutoff radius. This is the typical volume divergent behavior of $A d S_{5}$ as shown in 4,29, 30].

To compare these results with those for the KS action is necessary for both backgrounds to have the same physical temperature. This is implemented by requiring that the physical perimeter of the temporal directions match at the point of comparison:

$$
\beta_{b . h .} e^{z\left(u_{R}\right)-3 x\left(u_{R}\right)}=\beta_{K S} h^{-1 / 4}\left(\tau_{R}\right) .
$$

This equation allows to determine $\beta_{K S}$, since for the black hole, $\beta_{b . h}$. is determined by the absence of conical singularities (see equation (2.27)). However, we still need a way to relate $\tau_{R}$ and $u_{R}$. As can be seen from various examples discussed in [12, 15], in the UV the coordinate $u$ is related to the standard radial coordinate as $u \approx 1 / 4 r^{4}$; this is also true for the nonextremal D3 brane (see equation (2.20)) as well as for the KT solution, the b-deformed conifold [12], KS and the resolved conifold [18]. The relationship between the radial coordinate $\tau$ and the standard conifold radius is

$$
d r=\frac{\epsilon^{2 / 3}}{2^{1 / 6} 3^{1 / 2}} \frac{\sinh \tau}{(\sinh 2 \tau-2 \tau)^{1 / 3}} d \tau .
$$

In the limit of large values of $r$ we obtain:

$$
r \approx \frac{3^{1 / 2}}{2^{5 / 6}} \epsilon^{2 / 3} e^{\tau / 3}
$$

Thus, in equation (3.1) we use that

$$
\tau_{R} \approx \frac{1}{4} \ln \left(\epsilon^{-8} u_{R}^{-3}\right)-0.954771252+\delta \tau_{R}
$$

The correction $\delta \tau_{R}$ is required to account for the limitations of the numerical analysis to deal with actual asymptotic values. It is a function of $a$ and $\epsilon$ and we have found it to have the approximate form,

$$
\delta \tau_{R}=C(a) \epsilon^{1 / 5}
$$

Our strategy to establishing the existence of the transition is as follows. We assume that the transition takes place at a value of $\epsilon$ such that $a^{-8 / 3} \epsilon=1$, then we find the $C(a)$ that makes both actions equal. Following the order in table 1, we obtained that 

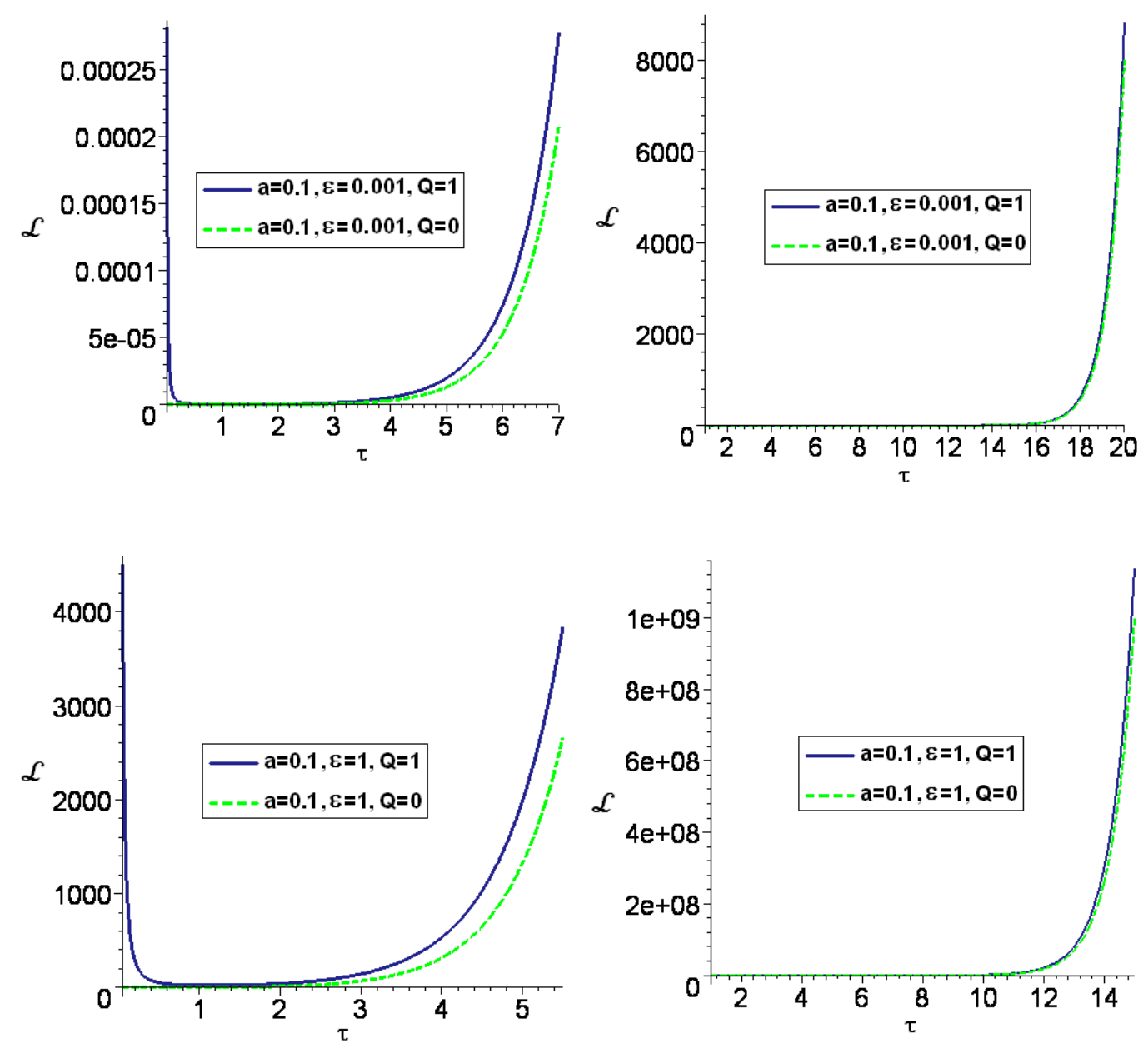

Figure 3: The KS Lagrangian in the IR and the UV for $\epsilon=0.001$ and $\epsilon=1$

$C(a)=\{-2.27,-3.264,-5.85,-9.8095\}$. We note that neither the factor $C(a)$ nor the power $1 / 5$ are solutions of a best fit problem but just values that give satisfactory results for all cases analyzed here. Our key observation then reduces to establish that the difference of actions changes sign appropriately around this point.

Let us address a technical issue. A problem to deal with here is the singularity in the KS background for $Q \neq 0$ (see in the appendix subsection A.1.1 for details). Fortunately, although conceptually an important point, the difference between the KS actions with and without $Q$ are qualitatively distinguishable only in the infrared regime (see for instance figures (3). In the UV regime, the leading asymptotic behavior is governed by $P$ and not $Q$.

The IR behavior is easy to understand. In this region, almost at the same value of $\tau$, the Lagrangian with $Q=0$ has a zero and the corresponding Lagrangian with $Q \neq 0$ has a minimum. We therefore switch at this point from the integrand with $Q \neq 0$ to the one with $Q=0$ as a regularization of the procedure. From the quantitative point of view, for $Q=0$ the infrared regime gives a negative contribution to the integral which increases 


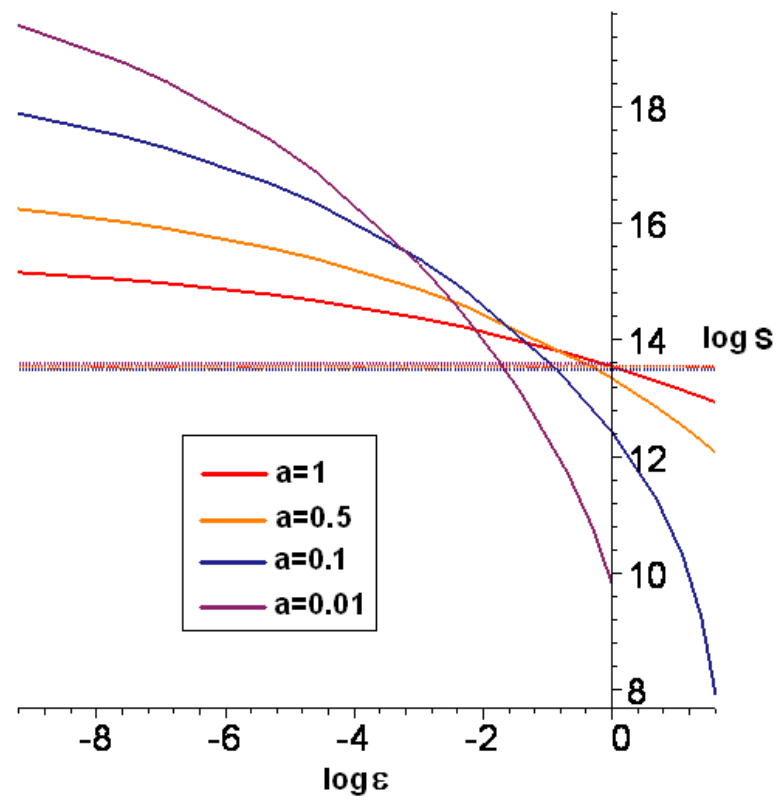

Figure 4: The Hawking-Page transition. The horizontal lines represent the values of the cascading black hole action for the four cases analyzed in this paper. The curves show the $\epsilon$-dependence of the corresponding KS actions.

slowly with $\epsilon$. Thus, for a fixed $a$, if for small $\epsilon$ the cascading black hole action dominates the difference of the actions, this is a reliable result because the negative contribution to the integral is negligible. On the other hand, if for large $\epsilon$ the KS action dominates, that is certainly true, because it does so in spite of the negative contribution to the integral.

Finally, the results of the actions comparison are presented in figure 4 . Curves represent the variation with $\epsilon$ of the KS action. Each curve with a given color must be compared with the horizontal line of the same color, i.e, with the cascading black hole with the corresponding temperature. As we can see, in each of these cases, the deconfined phase dominates for small values of $\epsilon$, while the confined phase dominates for large values of this parameter. The larger the value of $a$, the larger the value of $\epsilon$ for the phase transition to take place.

A limitation of the approach described here is that it works for relatively small values of $\epsilon$, implying relatively small values of $a$. Otherwise, the correction given by (3.5) might stop to being small. Moreover, the difference between the KS solutions with $Q=0$ and $Q \neq 0$ could contribute as well. Our calculations indicate that the transition can be detected for values as large as $\epsilon=10(a \sim 1)$.

\section{Some properties of a confining field theory at finite temperature via gauge/gravity duality}

\subsection{The running of the gauge couplings at finite temperature}

One of the most interesting properties of the cascading solutions is that they encode the running of a gauge coupling. In particular the matching of the supergravity modes with 
the two gauge couplings takes the form [10]:

$$
\begin{aligned}
\frac{4 \pi^{2}}{g_{1}^{2}}+\frac{4 \pi^{2}}{g_{2}^{2}} & =\frac{\pi}{g_{s} e^{\Phi}}, \\
\frac{4 \pi^{2}}{g_{1}^{2}}-\frac{4 \pi^{2}}{g_{2}^{2}} & =\frac{1}{g_{s} e^{\Phi}}\left[\frac{1}{2 \pi \alpha^{\prime}}\left(\int_{S^{2}} B_{2}\right)-\pi\right] .
\end{aligned}
$$

For the KT and KS solutions one finds a result that exactly matches the field theory value of the running coupling for a gauge theory with $\mathrm{SU}(N+M) \times \mathrm{SU}(N)$ gauge group.

For the cascading black hole solutions we can assume that a similar correspondence holds and evaluate $B_{2}$. The integral of $B_{2}$ over the appropriate two-cycle is proportional to the function $f(u)$ in (2.4). Since our solution is numerical we need first to find out what is the behavior of $f(u)$ in the UV regime. If we assume that as $u \rightarrow 0$,

$$
f(u) \approx-\frac{B}{4} P \mathrm{e}^{\Phi(u)} \ln (a u)+f_{r},
$$

where $f_{r}$ is a constant, then criterion (2.28) fixes

$$
B=\lim _{u_{\text {sing }} \rightarrow 0} 4 \frac{\mathrm{e}^{4\left(y_{0}+w_{0}\right)}}{\Phi_{1}+\frac{1}{u} \text { sing }} .
$$

The pole in this expression is expected to be cancelled by the logarithmic singularity in eq. (4.2). Substituting $B$ in (4.2), we compare the behavior of the resulting expression with

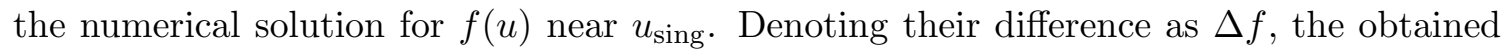
results are presented in figure (5) for all four cases in table 1 .

It can be seen, in the UV regime, that up to corrections well below $10^{-10}, f_{r}$ is indeed a constant. The approximated values of $f_{r}$ corresponding to the analyzed cases are $\{3.22186396,2.22309019,0.99140974,0.41584279\}$.

Thus, the running of the difference of the inverse squares of the coupling constants have several interesting properties. First, it is proportional to $P$ which is the parameter breaking the conformal invariance. More importantly, we have the following

$$
\ln (a u) \sim \ln \left(\frac{\rho_{0}^{4}}{\rho^{4}}\right) \sim \ln \left(\frac{T}{\Lambda}\right) .
$$

In the above expression we have used that $2 a=\rho_{0}^{4}$, where $\rho_{0}$ is the position of the horizon in the standard nonextremal D3-brane solution. Further $\rho_{0}=T \pi R^{2}$ for nonextremal D3 branes and finally $u \approx 1 / 4 \rho^{4}$ in the uv region.

Thus, we conclude that the gravity dual of the cascading black hole has

$$
\frac{1}{g_{1}^{2}}-\frac{1}{g_{2}^{2}} \sim P \ln \left(\frac{\Lambda}{T}\right)
$$

It is worth stressing that the high temperature behavior of the gauge coupling in YangMills theories depends on the renormalization schemes at finite $T$. There also seem to be some discrepancies between the imaginary and real time formalism. In a sense our result should be interpreted as the string theory prediction and it coincides with the general perturbative reasoning [25, 26]. 

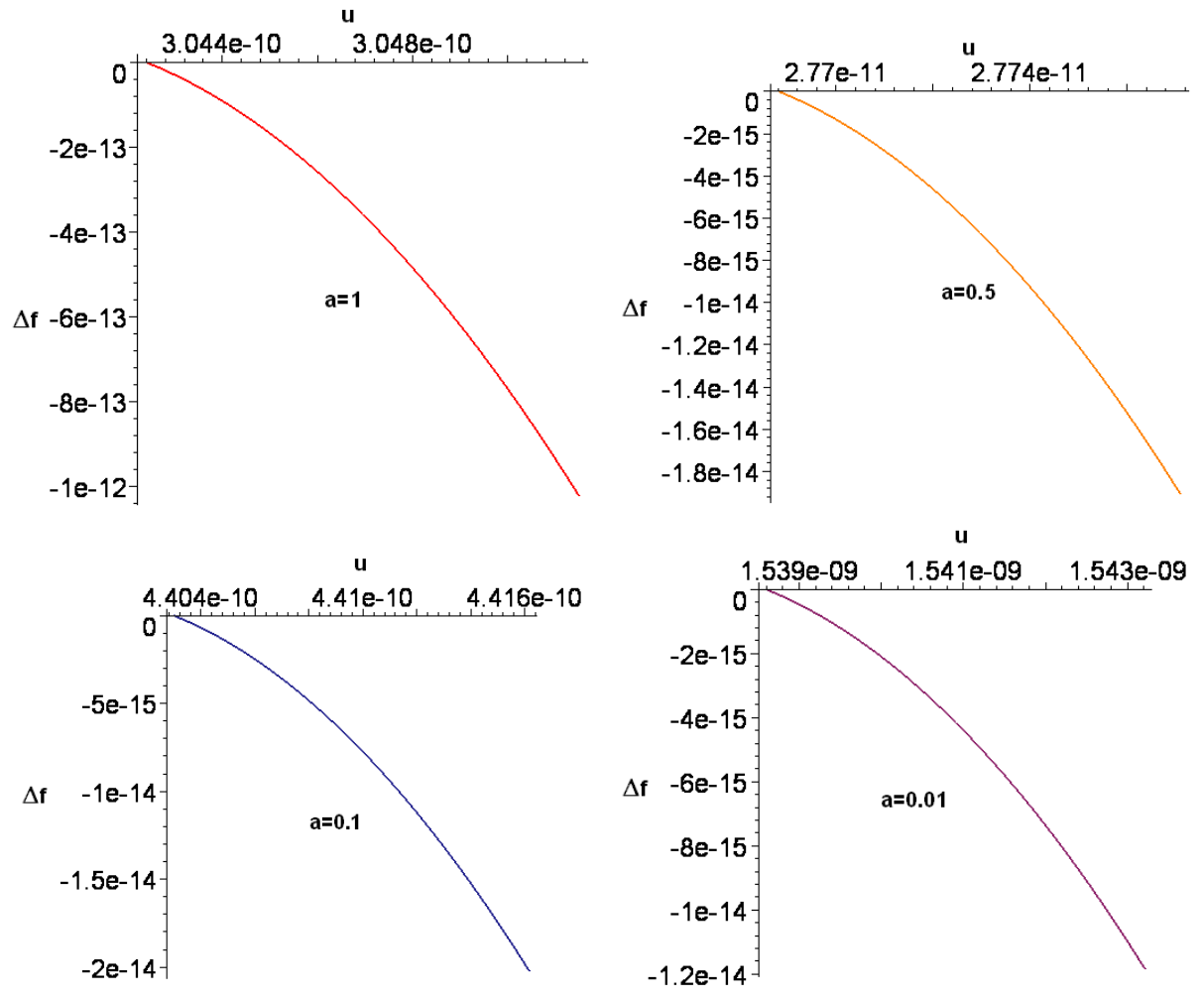

Figure 5: Behavior of $\Delta f$ for $a=1,0.5,0.1,0.01$. Since it is clearly below our 'zero' of $10^{-10}$, this shows that equation (4.2) represents very accurately the form of $f(u)$ in the UV-limit.

\subsection{The viscosity bound}

In this section we simply note that the solution [15] satisfies the condition of [27] which implies the viscosity bound. Namely, we find that for the metric (2.1) the Ricci tensor satisfies

$$
R_{0}^{0}-R_{1}^{1}=4 e^{2 z-10 y} \frac{d^{2} x(u)}{d u^{2}}
$$

This expression is identically zero due to the equation of motion of $x$.

In principle, it has been argued that the above expression is sufficient to guarantee the viscosity bound [27, 28]. We will nevertheless proceed to check the bound explicitly. The main reason being that our background is cascading and that might involve subtleties not considered in previous analysis and also that [27] used some nonconvariant counterterms. Since our solutions satisfy the criterion (2.23), we obtain for the shear diffusion constant,

$$
D=\frac{|\alpha-3|}{16 \pi} \frac{1}{T} \text {. }
$$

Assuming the black hole temperature to be given by (2.27), for the viscosity-entropy ratio,

$$
\frac{\eta}{s}=\frac{|\alpha-3|}{16 \pi} \text {. }
$$

In figure 6 we can observe that, for all the four cases in table 1, $\alpha \rightarrow-1$ from below as $u$ approach the horizon. In this figure the values of $u$ were conveniently rescaled for each 


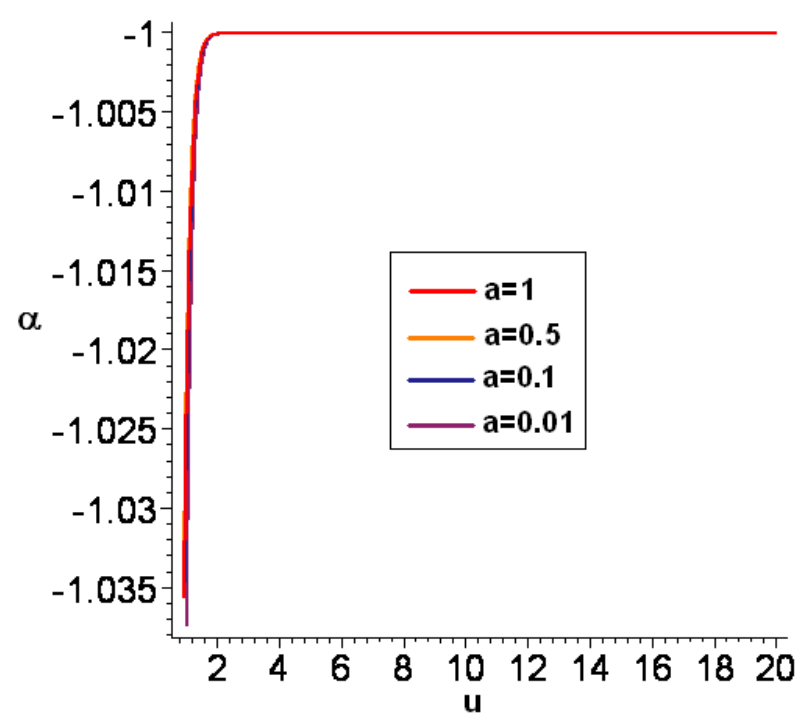

Figure 6: Behavior of the parameter $\alpha$ as $u \rightarrow u_{\text {hor }}$.

case using the ratio between the different values of $a$. The result seems to indicate that indeed the inequality is saturated. We must note here that, in all the cases we analyzed, the value to which $\alpha$ converges is actually slightly bigger than -1 . For these four cases we obtained $\{-0.999999937,-0.999999946,-0.999999977,-0.999999997\}$. Nevertheless, since the difference with respect to -1 is too close to our numerical 'zero' we regard this effect as a numerical artifact due to the proximity to the horizon.

\subsection{Drag force}

In this section we apply the analysis of [34, 35] to discuss the drag force on a quark moving in a strongly coupled plasma. We emphasize the general conditions for a supergravity background to admit the motion of a classical string that can be interpreted as a drag force on a moving quark. Our analysis mirrors the generality discussed in [36], however, here we do not assume AdS asymptotics, in particular we aim at understanding cascading backgrounds.

We consider here a five-dimensional subspace of a supergravity background dual to a field theory.

$$
d s^{2}=-G_{00} X_{0}^{2}+G_{x x}\left(d X_{1}^{2}+d X_{2}^{2}+d X_{3}^{2}\right)+G_{u u} d u^{2},
$$

where the metric components $G_{M N}$ are functions of the radial coordinate $u$ only. Following [34, 35], we assume the world-sheet to be embedded as $t=X_{0}$ and $\sigma=u$ and we allow for

$$
X_{1}=v t+\xi(u)
$$

which means that the end of the string is moving with velocity $v$. In the field theory side we interpret the end of the string as a quark moving with velocity $v$. The Nambu-Goto 
action is

$$
S=\frac{1}{2 \pi \alpha^{\prime}} \int d t d \sigma \sqrt{\left[G_{00}\left(G_{u u}+G_{x x} \xi^{2}\right)-G_{x x} G_{u u} v^{2}\right]}
$$

Since the action does not depend explicitly on $\sigma$, one has that the conjugate momenta $\frac{\partial L}{\partial \xi^{\prime}}$ is a constant, where $L$ is the Lagrangian,

$$
\Pi_{\xi}=\frac{\partial L}{\partial \xi^{\prime}}=-\frac{G_{00} G_{x x} \xi^{\prime}}{\sqrt{G_{00}\left(G_{u u}+G_{x x} \xi^{\prime 2}\right)-G_{u u} G_{x x} v^{2}}} .
$$

It can be rearranged to obtain

$$
\xi^{\prime}=\Pi_{\xi} \sqrt{\frac{G_{u u}\left(G_{00}-G_{x x} v^{2}\right)}{G_{00} G_{x x}\left(G_{00} G_{x x}-\Pi_{\xi}^{2}\right)}} .
$$

A key simplifying observation is that, since $\xi^{\prime}$ cannot be imaginary, we need both expressions in numerator and denominator to flip signs simultaneously. This fixes $\Pi_{\xi}$ to be

$$
\Pi_{\xi}^{2}=G_{00}(x) G_{x x}\left(u_{*}\right)
$$

Here, $u_{*}$ denotes the radial coordinate satisfying

$$
G_{00}\left(u_{*}\right)=G_{x x}\left(u_{*}\right) v^{2}
$$

The rate of change of momentum is calculated to be

$$
\frac{d p_{1}}{d t}=\sqrt{-g} T_{x_{1}}^{u}
$$

where

$$
T_{x_{1}}^{u}=-\frac{1}{2 \pi \alpha^{\prime}} G_{x_{1} \nu} g^{u \alpha} \phi_{\alpha} X^{\nu} .
$$

Here, $G_{i j}$ denotes the metric in (4.6), while $g_{i j}$ denotes the induced metric on the worldsheet. After some algebraic simplifications, we obtain

$$
\frac{d p_{1}}{d t}=-\frac{1}{2 \pi \alpha^{\prime}} \Pi_{\xi} .
$$

We can rewrite of above equation as

$$
\frac{d p_{1}}{d t}=-k p_{1},
$$

if condition (4.12) dictates $\Pi_{\xi}$ to be proportional to $p_{1}$. In the above equation, $k$ is the drag force constant. 
Conditions for drag force. Let us discuss the conditions under which we can observe a drag force proportional to the momentum. We need to solve for a radial position $u_{*}$ such that

$$
v^{2}=G_{00}\left(u_{*}\right) / G_{x x}\left(u_{*}\right)
$$

The expression for the canonical momentum is

$$
\Pi_{\xi}=v G_{x x}\left(u_{*}(v)\right)
$$

So, the question for a drag force essentially boils down to verifying that

$$
G_{x x}\left(u_{*}(v)\right)=\frac{b}{\sqrt{1-v^{2}}}
$$

where $b$ is a constant.

Two comments are in order about the relevant energy scales and the speed of the quarks:

- The nonrelativistic motion of the probe corresponding to small $v$ is localized near the points where $G_{00}$ vanishes. This corresponds, generically, to the horizon. In terms of energy scales, it corresponds to the infrared region.

- Relativistic velocities $v$ close to the speed of light correspond to radial positions for which $G_{00} \approx G_{x x}$. Generically, this is the asymptotic region, which in terms of the field theory is the ultraviolet region and corresponds to the near conformal limit.

Non-extremal D3 brane. In the case of non-extremal D3 branes we have that

$$
G_{00}=u^{2}\left(1-\frac{u_{0}^{4}}{u^{4}}\right), \quad G_{x x}=u^{2} .
$$

The equation that determines the position $u_{*}$ as a function of the velocity of the end of the string is thus:

$$
v^{2}=\frac{G_{00}}{G_{x x}}=\left(1-\frac{u_{0}^{4}}{u_{*}^{4}}\right)
$$

Thus, we find

$$
u_{*}^{2}=\frac{u_{0}^{2}}{\sqrt{1-v^{2}}}
$$

We can now easily verify that

$$
G_{x x}\left(u_{*}(v)\right)=u_{*}^{2}=\frac{u_{0}^{2}}{\sqrt{1-v^{2}}}
$$

This expression coincides with the criterion (4.19). This case was discussed in refs. 34, 35]. 
Cascading black holes. Here we simply adjust the computation of the drag force presented, for example, in [34, 35]. The calculations presented here were, in particular, performed in 37, 38] without using the full metric of the cascading black hole. The AdS part of the metric in consideration in string frame is

$$
d s^{2}=e^{\Phi / 2}\left[e^{2 z}\left(-e^{-6 x} d X_{0}^{2}+e^{2 x} d X_{i} d X^{i}\right)+e^{10 y-2 z} d u^{2}\right]
$$

We consider the worldsheet to be along $t=X_{0}$ and $\sigma=u$ directions. Following the general arguments at the beginning of this subsection

$$
\Pi_{\xi}=\sqrt{v} e^{\frac{\Phi}{2}+2 z}(u(x(v)))
$$

Here, $u$ is chosen to satisfy $v=e^{-4 x(u)}$ for a given $v$. This relation is to be viewed as an equation for the radial coordinate $u$, it defines a particular value $u_{p}$. We need to evaluate relation (4.25) at $u=u_{p}$ and, in turn, find $\Pi_{\xi}$ as a function of $v$. Further, to establish the existence of a drag force we need to verify the existence of a $b$ such that

$$
\Pi_{\xi}=b \frac{v}{\sqrt{\left(1-v^{2}\right)}}
$$

which thus implies that $\Pi_{\xi}=\frac{b}{m} p_{1}$ and directly leads to a drag force parameter using equations (4.15) and (4.16). With this aim, equating equations (4.25) and (4.26) we obtain,

$$
\begin{aligned}
& b(u)=\sqrt{2} \mathrm{e}^{\frac{\Phi}{2}+2 z} \sqrt{\sinh (4 a u)} \\
& v(u)=\mathrm{e}^{-4 a u} .
\end{aligned}
$$

First, note that in the IR regime $(u \rightarrow \infty)$,

$$
b \rightarrow \mathrm{e}^{\frac{\Phi(u)}{2}} \mathrm{e}^{2 z_{*}} \mathrm{e}^{2(\alpha+1) a u},
$$

where we used the asymptotical expression (2.22) for $z(u)$. In the pure D3 case, where $\Phi(u)=0$ and $\alpha=-1$, we have that $b=\exp \left(2 z_{*}\right)$. Recalling that, as shown in subsection 1.2, for our solution $\alpha \approx-1$, and noting that the dilaton is very weak near the horizon (for instance, for our four cases we obtained $\Phi_{*} \approx\left\{-3 \times 10^{-7},-2 \times 10^{-7},-1.2 \times\right.$ $\left.10^{-7},-1.4 \times 10^{-8}\right\}$ ), we see that for the cascading black hole $b \approx$ constant in the IR-limit.

On the other hand, knowing that as $u \rightarrow 0, z(u)$ behaves as described by eq. (2.29), we obtain,

$$
b \rightarrow \sqrt{2 a} \mathrm{e}^{\frac{\Phi_{0}}{2}},
$$

implying that $b$ is finite in the UV-limit.

This way, we have shown analytically that when the string explores any of the two asymptotic regions, we verify the existence of a drag force. For a given temperature the variability of $b$ from one regime to the other is mainly related to the dilaton variability. In figure 7 we show the drag force coefficient $b$ as given by eqs. (4.27) for all values of $v$, that is, for all velocities of the external quarks. The curves represent the four cases in table 1 . It is worth stressing that in the relativistic regime there is a clear velocity dependence in the coefficient $b$. However, for the range of values of temperatures that we have explored it, it does not seem to be universal. In particular, the figure 7 shows that in the regime of relativistic velocities some curves have inflections upwards while other bend downward. Clearly, it seems to depend on the temperature and deserves further study. 


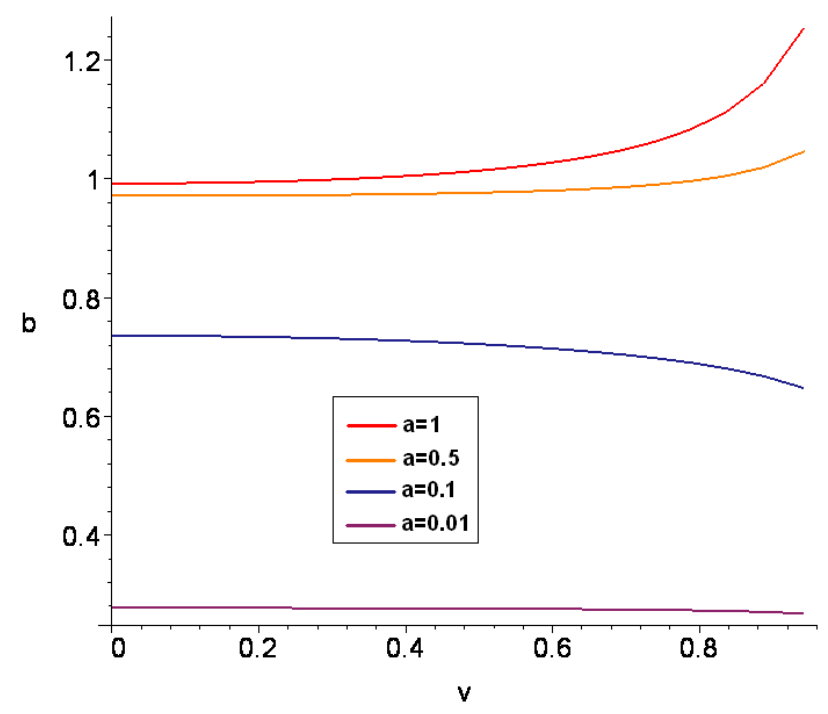

Figure 7: Behavior of the drag force parameter $b$ as a function of the quark velocity $v$.

\section{Discussion and outlook}

In this paper we have established numerically the existence of a Hawking-Page phase transition between the supergravity solution of [15] which represents a black hole in a cascading background and the Klebanov-Strassler solution.

We also studied some properties of the deconfined phase given by our cascading black hole solution. For instance, we have found that the difference of the inverse squares of the coupling constants is proportional to $P$ (which is the parameter breaking the conformal invariance) and that it runs as $\ln (\Lambda / T)$.

We also verify that our solution seems to satisfy the well-known bound for the viscosity to entropy ratio.

Finally, we have explicitly shown that the universal form of the horizon in this theory guarantees the existence of a drag force for a fundamental string moving in this background and in the dual field theory that can be interpreted as jet quenching. The near horizon region describes nonrelativistic motion of the quarks and since the near horizon geometry has been shown to be universal we conclude that the nonrelativistic drag force on the quarks is also universal in the class of cascading theories. In the relativistic region we also found a well-defined drag force parameter. Numerically, we observed a velocity dependence for this parameter in the relativistic region.

It is important to stress that most of the calculations performed in this paper requires knowing the solution for all values of the radius and that an analysis solely based on the asymptotic near the horizon is not reliable.

Let us conclude with listing a number of possible directions. Cascading theories, along the lines of the particular model described here are generic in the AdS/CFT. In fact, we believe that there might be infinite classes of them based on some results presented in [39, 40]. The fact that the cone over $Y^{p, q}$ does not admit complex deformations seems like a deterrent for a construction directly mimicking the warped deformed conifold of 
Klebanov and Strassler but a more general solution where the fluxes play a crucial role are not ruled out. Turning on temperature of these solutions should not change substantially the asymptotic UV form obtained in [39, 40].

It would be interesting to better understand the charges in the comparison between the cascading black hole with the KS background. Basically the KS solution has $Q=0$, that is, the number of D3 branes at the tip deformed conifold equal to zero. As shown in [9] and reviewed in section A.1.1, we see that nonzero $Q$ results in a singularity at the origin. Another interesting direction would be the construction of black holes in the backgrounds with back reacted flavor such like the one described in ref. [41]. In particular, some of these backgrounds should be natural generalizations of the construction of [15].

While this manuscript was in the final stage of its preparation, a new paper was posted where the authors claim to have found evidence for a transition in a solution with a black hole in the IR and $\mathrm{KT}$ in the UV [42]. We plan an in-depth discussion of the relation between our and their solutions. Preliminarily, let us note here that there are some key differences between our solutions. First, as far as we understand, to find their solution they need to numerically look for a zero of a function in ten variables, i.e., the norm of their 'mismatch vector'. Since this is a positive semi-definite function, assuming that this zero exists, this problem can be translated into finding the global minimum of a 10D-surface. Nevertheless, this is a case of constrained optimization, since the Hamiltonian constraint is also valid in ten dimensions, where it shows as a reparametrization of the radial coordinate. However, the authors of [42] made no mention to whether their numerical solution satisfies this constraint. As opposed to that, our solutions are forced to automatically satisfy the zero-energy constraint and that this certainly happen is one of the main criteria of quality we use to certify our numerical output. This was first explained in ref. [15] and emphasized in this manuscript. Secondly, their solution seems to have exactly KT in the boundary (we note here that the KT solution does not satisfy the Hamiltonian constraint for $a \neq 0$ ) and to erase the large $u$ singularity typical of KT (we called this singular point as $u_{\text {sing }}$ ), pasting the black hole metric in the IR solution somewhere in the 'middle' of the radial domain. Our solution extents from $u_{\text {sing }}$ to infinity and, as explained in section 2.4, we set $u_{\text {sing }}=0$ to guarantee the completeness of our background.

\section{Acknowledgments}

We thank Alex Buchel for various comments, Elena Cáceres for comments on the drag force and Javier Mas for various useful comments and explanations about related matters. This work is partially supported by Department of Energy under grant DE-FG02-95ER40899 to the University of Michigan and by grant CNPq/CLAF-150548/2004-4. C. T-E thanks the MCTP for hospitality during the final stages of this work and LPZ thanks Universidad de Santiago de Compostela for a very warm hospitality. 


\section{A. The Klebanov-Strassler background}

The KS metric is of the form:

$$
\begin{aligned}
d s_{10}^{2}= & h^{-1 / 2}(\tau) d x_{n} d x_{n}+h^{1 / 2}(\tau) d s_{6}^{2} \\
d s_{6}^{2}= & \frac{1}{2} \varepsilon^{4 / 3} K(\tau)\left[\frac{1}{3 K^{3}(\tau)}\left(d \tau^{2}+\left(g^{5}\right)^{2}\right)\right. \\
& \left.+\cosh ^{2}\left(\frac{\tau}{2}\right)\left[\left(g^{3}\right)^{2}+\left(g^{4}\right)^{2}\right]+\sinh ^{2}\left(\frac{\tau}{2}\right)\left[\left(g^{1}\right)^{2}+\left(g^{2}\right)^{2}\right]\right],
\end{aligned}
$$

the one-forms $g^{i}$ are defined as

$$
\begin{array}{ll}
g^{1}=\frac{e^{1}-e^{3}}{\sqrt{2}}, & g^{2}=\frac{e^{2}-e^{4}}{\sqrt{2}}, \\
g^{3}=\frac{e^{1}+e^{3}}{\sqrt{2}}, & g^{4}=\frac{e^{2}+e^{4}}{\sqrt{2}} \\
g^{5}=e^{5} &
\end{array}
$$

where

$$
\begin{aligned}
e^{1} & \equiv-\sin \theta_{1} d \phi_{1}, \quad e^{2} \equiv d \theta_{1}, \\
e^{3} & \equiv \cos \psi \sin \theta_{2} d \phi_{2}-\sin \psi d \theta_{2}, \\
e^{4} & \equiv \sin \psi \sin \theta_{2} d \phi_{2}+\cos \psi d \theta_{2}, \\
e^{5} & \equiv d \psi+\cos \theta_{1} d \phi_{1}+\cos \theta_{2} d \phi_{2} .
\end{aligned}
$$

The function in the metric is

$$
\mathcal{K}(\tau)=\frac{(\sinh (2 \tau)-2 \tau)^{1 / 3}}{2^{1 / 3} \sinh \tau} .
$$

For asymptotically large values of $\tau$ one can introduce a natural radial coordinate $r^{2}=$ $\frac{3}{2^{5 / 3}} \varepsilon^{4 / 3} e^{2 \tau / 3}$ such that the metric on the conifold becomes $d s_{6}^{2} \approx d r^{2}+r^{2} d s_{T^{1,1}}^{2}$.

The matter fields are as follow:

$$
\begin{gathered}
F_{3}=\frac{M \alpha^{\prime}}{2}\left\{g^{5} \wedge g^{3} \wedge g^{4}+d\left[F(\tau)\left(g^{1} \wedge g^{3}+g^{2} \wedge g^{4}\right)\right]\right\} \\
=\frac{M \alpha^{\prime}}{2}\left\{g^{5} \wedge g^{3} \wedge g^{4}(1-F)+g^{5} \wedge g^{1} \wedge g^{2} F\right. \\
\left.+F^{\prime} d \tau \wedge\left(g^{1} \wedge g^{3}+g^{2} \wedge g^{4}\right)\right\}
\end{gathered}
$$

with $F(0)=0$ and $F(\infty)=1 / 2$, and

$$
\begin{gathered}
B_{2}=\frac{g_{s} M \alpha^{\prime}}{2}\left[f(\tau) g^{1} \wedge g^{2}+k(\tau) g^{3} \wedge g^{4}\right] \\
H_{3}=d B_{2}=\frac{g_{s} M \alpha^{\prime}}{2}\left[d \tau \wedge\left(f^{\prime} g^{1} \wedge g^{2}+k^{\prime} g^{3} \wedge g^{4}\right)+\frac{1}{2}(k-f) g^{5} \wedge\left(g^{1} \wedge g^{3}+g^{2} \wedge g^{4}\right)\right] .
\end{gathered}
$$

The self-dual 5 -form field strength may be decomposed as $\tilde{F}_{5}=\mathcal{F}_{5}+\star \mathcal{F}_{5}$. We have

$$
\mathcal{F}_{5}=B_{2} \wedge F_{3}=\frac{g_{s} M^{2}\left(\alpha^{\prime}\right)^{2}}{4} \ell(\tau) g^{1} \wedge g^{2} \wedge g^{3} \wedge g^{4} \wedge g^{5}
$$


where

$$
\ell=f(1-F)+k F
$$

and

$$
\begin{aligned}
\star \mathcal{F}_{5} & =4 g_{s} M^{2}\left(\alpha^{\prime}\right)^{2} \varepsilon^{-8 / 3} d x^{0} \wedge d x^{1} \wedge d x^{2} \wedge d x^{3} \wedge d \tau \frac{\ell(\tau)}{K^{2} h^{2} \sinh ^{2}(\tau)} . \\
\alpha & =4\left(g_{s} M \alpha^{\prime}\right)^{2} \varepsilon^{-8 / 3} .
\end{aligned}
$$

The solution for the functions defining the matter content is:

$$
\begin{aligned}
F(\tau) & =\frac{\sinh \tau-\tau}{2 \sinh \tau}, \\
f(\tau) & =\frac{\tau \operatorname{coth} \tau-1}{2 \sinh \tau}(\cosh \tau-1), \\
k(\tau) & =\frac{\tau \operatorname{coth} \tau-1}{2 \sinh \tau}(\cosh \tau+1), \\
\ell(\tau) & =f(1-F)+k F=\frac{\tau \operatorname{coth} \tau-1}{4 \sinh ^{2} \tau}(\sinh 2 \tau-2 \tau) .
\end{aligned}
$$

The warp factor with the condition that it vanishes at infinity is

$$
h(\tau)=\alpha \frac{2^{2 / 3}}{4} I(\tau)=\left(g_{s} M \alpha^{\prime}\right)^{2} 2^{2 / 3} \varepsilon^{-8 / 3} I(\tau),
$$

where

$$
I(\tau) \equiv \int_{\tau}^{\infty} d x \frac{x \operatorname{coth} x-1}{\sinh ^{2} x}(\sinh (2 x)-2 x)^{1 / 3} .
$$

Two important limits are:

$$
\begin{aligned}
I(\tau \rightarrow 0) & \rightarrow a_{0}+O\left(\tau^{2}\right) \\
I(\tau \rightarrow \infty) & \rightarrow 3 \cdot 2^{-1 / 3}\left(\tau-\frac{1}{4}\right) e^{-4 \tau / 3},
\end{aligned}
$$

where $a_{0} \approx 0.71805$. At large $\tau$ the integrand becomes

$$
h \approx 3^{4} 2^{-4} \frac{\left(g_{s} M \alpha^{\prime}\right)^{2}}{r^{4}} \ln \left(\frac{2^{5 / 3}}{3} \frac{r^{2}}{\epsilon^{4 / 3}}\right),
$$

where we have used that for large radius $r^{2}=\frac{3}{2^{5 / 3}} \varepsilon^{4 / 3} e^{2 \tau / 3}$ :

\section{A.1 The action for the KS model}

A convenient way to look at this solution is through the prism of a one-dimensional system as discussed in [18]. Motivated by the form of the deformed conifold metric we make the following ansatz for the metric

$$
d s^{2}=e^{2 p-x}\left(e^{2 A} d x^{\mu} d x^{\mu}+d u^{2}\right)+\left[e^{-6 p-x} g_{5}^{2}+e^{x+y}\left(g_{1}^{2}+g_{2}^{2}\right)+e^{x-y}\left(g_{3}^{2}+g_{4}^{2}\right)\right] .
$$


The ansatz for the p-forms is:

$$
\begin{aligned}
H_{3} & =d u \wedge\left[f^{\prime}(u) g_{1} \wedge g_{2}+k^{\prime}(u) g_{3} \wedge g_{4}\right] \\
F_{3} & =F(u) g_{1} \wedge g_{2} \wedge g_{5}+[2 P-F(u)] g_{3} \wedge g_{4} \wedge g_{5}+F^{\prime}(u) d u \wedge\left(g_{1} \wedge g_{3}+g_{2} \wedge g_{4}\right), \\
F_{5} & =\mathcal{F}_{5}+\mathcal{F}_{5}^{*}, \quad \mathcal{F}_{5}=K(u) g_{1} \wedge g_{2} \wedge g_{3} \wedge g_{4} \wedge g_{5} \\
K(u) & \equiv Q+k(u) F(u)+f(u)[2 P-F(u)]
\end{aligned}
$$

where $F, f, k$ are functions to be determined and $P$ and $Q$ are constants. We explicitly ensure that the Bianchi identities for the p-forms are satisfied automatically. The 1-d action reproducing the resulting equations of motion restricted to the above ansatz has the following general structure

$$
S=c \int d u e^{4 A}\left[3 A^{\prime 2}-\frac{1}{2} G_{a b}(\varphi) \varphi^{\prime a} \varphi^{\prime b}-V(\varphi)\right],
$$

where $c=-4 \frac{V o l_{9}}{2 \kappa_{10}^{2}}$. It should be supplemented with the "zero-energy" constraint

$$
3 A^{\prime 2}-\frac{1}{2} G_{a b}(\varphi) \varphi^{\prime a} \varphi^{\prime b}+V(\varphi)=0 .
$$

The action (A.23) is thus a classical mechanical action for the fields $A$ and $\varphi^{a}=$ $(x, y, p, \Phi, f, k, F)$. The corresponding kinetic and potential terms in (A.23) are found to be:

$$
\begin{aligned}
G_{a b}(\varphi) \varphi^{\prime a} \varphi^{\prime b}= & x^{\prime 2}+\frac{1}{2} y^{\prime 2}+6 p^{\prime 2}+\frac{1}{4}\left[\Phi^{\prime 2}+e^{-\Phi-2 x}\left(e^{-2 y} f^{\prime 2}+e^{2 y} k^{\prime 2}\right)+2 e^{\Phi-2 x} F^{\prime 2}\right] \\
V(\varphi)= & \frac{1}{4} e^{-4 p-4 x}-e^{2 p-2 x} \cosh y+\frac{1}{4} e^{8 p} \sinh ^{2} y \\
& +\frac{1}{8} e^{8 p}\left[\hat{a} e^{-\Phi-2 x}(f-k)^{2}+e^{\Phi-2 x}\left[e^{-2 y} F^{2}+e^{2 y}(2 P-F)^{2}\right]+e^{-4 x} K^{2}\right]
\end{aligned}
$$

where $K$ is the combination of the independent functions $f, k, F$ given in (A.22).

The first order equations for the independent functions $A, x, y, p, f, k, F, \Phi$ are:

$$
\begin{aligned}
x^{\prime} & =-e^{-2 p-2 x}-\hat{a} e^{4 p-2 x} K, \quad y^{\prime}=e^{4 p} \sinh y, \\
p^{\prime} & =\frac{1}{3} e^{4 p} \cosh y-\frac{1}{6} e^{-2 p-2 x}+\frac{1}{6} e^{4 p-2 x} K \\
A^{\prime} & =-\frac{1}{3} e^{4 p} \cosh y-\frac{1}{3} e^{-2 p-2 x}-\frac{1}{6} e^{4 p-2 x} K \\
f^{\prime} & =e^{\Phi+4 p+2 y}(2 P-F), \quad k^{\prime}=e^{\Phi+4 p-2 y} F, \quad F^{\prime}=-\frac{1}{2} e^{-\Phi+4 p}(f-k), \quad \Phi^{\prime}=0 .
\end{aligned}
$$

The functions that we introduced in this subsection are explicitly given in terms of the solution of the previous subsection as:

$$
\begin{aligned}
\mathrm{e}^{x} & =\frac{1}{4} \epsilon^{4 / 3} \mathcal{K} \sinh (\tau) h^{1 / 2}, \\
\mathrm{e}^{2 p} & =24^{1 / 3} h^{-1 / 3} \epsilon^{-8 / 9} \mathcal{K}^{1 / 3} \sinh (\tau)^{-1 / 3}, \\
\mathrm{e}^{y} & =\tanh \left(\frac{\tau}{2}\right) .
\end{aligned}
$$


We will also use explicit forms for the derivative with respect to $u$

$$
\begin{aligned}
A^{\prime} & =-\frac{1}{6}\left(\mathrm{e}^{4 p}\left(\mathrm{e}^{y}+\mathrm{e}^{-y}\right)+2 \mathrm{e}^{-2 p} \mathrm{e}^{-2 x}+K \mathrm{e}^{4 p} \mathrm{e}^{-2 x}\right), \\
p^{\prime} & =\frac{1}{6}\left(\mathrm{e}^{4 p}\left(\mathrm{e}^{y}+\mathrm{e}^{-y}\right)-\mathrm{e}^{-2 p} \mathrm{e}^{-2 x}+K \mathrm{e}^{4 p} \mathrm{e}^{-2 x}\right), \\
y^{\prime} & =\mathrm{e}^{4 p} \sinh \left(\ln \left(\mathrm{e}^{y}\right)\right), \quad x^{\prime}=-\mathrm{e}^{-2 p} \mathrm{e}^{-2 x}-\frac{K}{2} \mathrm{e}^{4 p} \mathrm{e}^{-2 x}, \quad \Phi=\Phi^{\prime}=0, \\
k^{\prime} & =F \mathrm{e}^{\Phi} \mathrm{e}^{4 p} \mathrm{e}^{-2 y}, \quad f^{\prime}=(2 P-F) \mathrm{e}^{\Phi} \mathrm{e}^{4 p} \mathrm{e}^{2 y}, \quad F^{\prime}=-\frac{f-k}{2} \mathrm{e}^{-\Phi} \mathrm{e}^{4 p} .
\end{aligned}
$$

The expression for the action we need to evaluate is finally of the form:

$$
\mathcal{L}=-\frac{4}{h} \mathrm{e}^{2 x} \mathrm{e}^{-4 p}\left(3 A^{\prime 2}-\frac{1}{2} G_{a b}-V\right), \quad S_{K S}=\int d \tau \mathcal{L} .
$$

\section{A.1.1 The KS background has no regular D3 branes at the apex}

As noted in [9], the KS-like Ansatz allows for general solutions having $K(u)$ with arbitrary $Q$ in (A.22). In the more standard literature $Q$ is identified as $N$. These solutions are, however, singular. Namely, near the apex of the deformed conifold (small values of $\tau$ ) the warp factor takes the form:

$$
h \approx \frac{Q}{\tau} .
$$

An alternative way of understanding this singularity is by realizing that it corresponds to the freedom to add a homogeneous solution to the warp factor. Namely, a solution of the form

$$
\frac{1}{\sqrt{g_{6}}} \partial_{\tau}\left(\sqrt{g_{6}} g^{\tau \tau} \partial_{\tau} \tilde{h}\right)=0
$$

or

$$
\tilde{h}=Q \int \frac{d \tau}{\mathcal{K}^{2} \sinh ^{2} \tau} .
$$

This last statement allows to interpret the elimination of the singularity as a statement about regular D3 branes. It is possible that, as in the interpretation for the resolved conifold of [18], this divergence signals a smearing of D3 branes along a three-dimensional subspace and therefore could also be cure, not just by turning off the charge $Q$, but also by localizing the branes appropriately as it was done recently in ref. [33].

This situation presents a conceptual problem. It is natural to compare a field theory with parameters $\left(N, M, \epsilon \sim \Lambda_{\text {strong}}\right)$ with a theory with $(N, M, a \sim$ Temperature $)$. In realistic cases, the scale $\Lambda_{\text {strong }}$ is dynamically generated but supergravity methods are still far from achieving that. However, for KS we have $N=0$ and for the cascading black hole we have $u_{\text {sing }}=0$. The comparison is really not completely clear. We deal with this point while analyzing the transition in the next section.

\section{References}

[1] J.M. Maldacena, The large- $N$ limit of superconformal field theories and supergravity, Adv. Theor. Math. Phys. 2 (1998) 231 Int. J. Theor. Phys. 38 (1999) 1113 hep-th/9711200. 
[2] O. Aharony, S.S. Gubser, J.M. Maldacena, H. Ooguri and Y. Oz, Large- $N$ field theories, string theory and gravity, Phys. Rept. 323 (2000) 183 hep-th/9905111.

[3] S.S. Gubser, I.R. Klebanov and A.W. Peet, Entropy and temperature of black 3-branes, Phys. Rev. D 54 (1996) 3915 hep-th/9602135;

I.R. Klebanov and A.A. Tseytlin, Entropy of near-extremal black p-branes, Nucl. Phys. B 475 (1996) 164 hep-th/9604089;

S.S. Gubser, I.R. Klebanov and A.A. Tseytlin, Coupling constant dependence in the thermodynamics of $N=4$ supersymmetric Yang-Mills theory, Nucl. Phys. B 534 (1998) 202 hep-th/9805156.

[4] E. Witten, Anti-de Sitter space, thermal phase transition and confinement in gauge theories, Adv. Theor. Math. Phys. 2 (1998) 505 hep-th/9803131.

[5] I.R. Klebanov and E. Witten, Superconformal field theory on threebranes at a Calabi-Yau singularity, Nucl. Phys. B 536 (1998) 199 hep-th/9807080.

[6] S.S. Gubser and I.R. Klebanov, Baryons and domain walls in an $N=1$ superconformal gauge theory, Phys. Rev. D 58 (1998) 125025 hep-th/9808075.

[7] I.R. Klebanov and N.A. Nekrasov, Gravity duals of fractional branes and logarithmic $R G$ flow, Nucl. Phys. B 574 (2000) 263 hep-th/9911096.

[8] I.R. Klebanov and A.A. Tseytlin, Gravity duals of supersymmetric $\mathrm{SU}(N) \times \mathrm{SU}(N+M)$ gauge theories, Nucl. Phys. B 578 (2000) 123 hep-th/0002159.

[9] I.R. Klebanov and M.J. Strassler, Supergravity and a confining gauge theory: duality cascades and $\chi S B$-resolution of naked singularities, JHEP 08 (2000) 052 hep-th/0007191.

[10] C.P. Herzog, I.R. Klebanov and P. Ouyang, D-branes on the conifold and $N=1$ gauge/gravity dualities, hep-th/020510d.

[11] A. Buchel, Finite temperature resolution of the Klebanov-Tseytlin singularity, Nucl. Phys. B 600 (2001) 219 hep-th/0011146.

[12] A. Buchel, C.P. Herzog, I.R. Klebanov, L.A. Pando Zayas and A.A. Tseytlin, Non-extremal gravity duals for fractional D3-branes on the conifold, JHEP 04 (2001) 033 hep-th/0102105.

[13] S.S. Gubser, C.P. Herzog, I.R. Klebanov and A.A. Tseytlin, Restoration of chiral symmetry: a supergravity perspective, JHEP 05 (2001) 028 hep-th/0102172.

[14] O. Aharony, A. Buchel and A. Yarom, Holographic renormalization of cascading gauge theories, Phys. Rev. D 72 (2005) 066003 hep-th/0506002.

[15] L.A. Pando Zayas and C.A. Terrero-Escalante, Black holes with varying flux: a numerical approach, JHEP 09 (2006) 051 hep-th/0605170].

[16] D. Marolf, Chern-Simons terms and the three notions of charge, hep-th/0006117.

[17] A. Buchel, Transport properties of cascading gauge theories, Phys. Rev. D 72 (2005) 106002 hep-th/0509083.

[18] L.A. Pando Zayas and A.A. Tseytlin, 3-branes on resolved conifold, JHEP 11 (2000) 028 hep-th/0010088.

[19] O. Aharony, J. Sonnenschein and S. Yankielowicz, A holographic model of deconfinement and chiral symmetry restoration, Ann. Phys. (NY) 322 (2007) 1420 hep-th/0604161. 
[20] D. Mateos, R.C. Myers and R.M. Thomson, Holographic phase transitions with fundamental matter, Phys. Rev. Lett. 97 (2006) 091601 hep-th/0605046.

[21] P. Benincasa and A. Buchel, Hydrodynamics of Sakai-Sugimoto model in the quenched approximation, Phys. Lett. B 640 (2006) 108 hep-th/0605076.

[22] A. Parnachev and D.A. Sahakyan, Chiral phase transition from string theory, Phys. Rev. Lett. 97 (2006) 111601 hep-th/0604173.

[23] T. Albash, V.G. Filev, C.V. Johnson and A. Kundu, A topology-changing phase transition and the dynamics of flavour, hep-th/0605088.

[24] L.A. Pando Zayas, Confinement/deconfinement transition in AdS/CFT, http://www.perimeterinstitute.ca/en/Events/View_Lectures/.

[25] J.I. Kapusta and C. Gale, Finite-temperature field theory principles and applications, Cambridge University Press, Cambridge U.K. (2006).

[26] M. Bellac, Thermal field theory, Cambridge University Press, Cambridge U.K. (1996).

[27] A. Buchel, On universality of stress-energy tensor correlation functions in supergravity, Phys. Lett. B 609 (2005) 392 hep-th/0408095.

[28] A. Buchel and J.T. Liu, Universality of the shear viscosity in supergravity, Phys. Rev. Lett. 93 (2004) 090602 hep-th/0311175.

[29] C.P. Herzog, A holographic prediction of the deconfinement temperature, Phys. Rev. Lett. 98 (2007) 091601 hep-th/0608151.

[30] C.A. Ballon Bayona, H. Boschi-Filho, N.R.F. Braga and L.A. Pando Zayas, On a holographic model for confinement/deconfinement, arXiv:0705.1529.

[31] R.-G. Cai and J.P. Shock, Holographic confinement/deconfinement phase transitions of $A d S / Q C D$ in curved spaces, arXiv:0705.3388.

[32] R.-G. Cai and N. Ohta, Deconfinement transition of $A d S / Q C D$ at $\mathcal{O}\left(\alpha^{\prime 3}\right)$, arXiv:0707.2013.

[33] I.R. Klebanov and A. Murugan, Gauge/gravity duality and warped resolved conifold, JHEP 03 (2007) 042 hep-th/0701064.

[34] C.P. Herzog, A. Karch, P. Kovtun, C. Kozcaz and L.G. Yaffe, Energy loss of a heavy quark moving through $N=4$ supersymmetric Yang-Mills plasma, JHEP 07 (2006) 013 hep-th/0605158.

[35] S.S. Gubser, Drag force in AdS/CFT, Phys. Rev. D 74 (2006) 126005 hep-th/0605182.

[36] C.P. Herzog, Energy loss of heavy quarks from asymptotically AdS geometries, JHEP 09 (2006) 032 hep-th/0605191.

[37] A. Buchel, On jet quenching parameters in strongly coupled non-conformal gauge theories, Phys. Rev. D 74 (2006) 046006 hep-th/060517g.

[38] E. Caceres and A. Guijosa, On drag forces and jet quenching in strongly coupled plasmas, JHEP 12 (2006) 068 hep-th/0606134.

[39] C.P. Herzog, Q.J. Ejaz and I.R. Klebanov, Cascading RG flows from new Sasaki-Einstein manifolds, JHEP 02 (2005) 009 hep-th/0412193. 
[40] B.A. Burrington, J.T. Liu, M. Mahato and L.A. Pando Zayas, Towards supergravity duals of chiral symmetry breaking in Sasaki-Einstein cascading quiver theories, JHEP 07 (2005) 019 hep-th/0504155.

[41] F. Benini, F. Canoura, S. Cremonesi, C. Núñez and A.V. Ramallo, Backreacting flavors in the Klebanov-Strassler background, arXiv:0706.1233; Unquenched flavors in the Klebanov-Witten model, JHEP 02 (2007) 090 hep-th/0612118.

[42] O. Aharony, A. Buchel and P. Kerner, The black hole in the throat - thermodynamics of strongly coupled cascading gauge theories, arXiv:0706.1768. 